Itinéraires Itinéraires

Littérature, textes, cultures

2017-2 | 2018

Féminismes quatrième génération

\title{
Faire naître et mourir les vagues : comment s'écrit l'histoire des féminismes
}

How Waves Arise and Die Out: The Writing of the History of Feminism

Bibia Pavard

\section{OpenEdition}

1 Journals

Édition électronique

URL : http://journals.openedition.org/itineraires/3787

DOI : $10.4000 /$ itineraires.3787

ISSN : 2427-920X

Éditeur

Pléiade

Référence électronique

Bibia Pavard, « Faire naître et mourir les vagues : comment s'écrit l'histoire des féminismes »,

Itinéraires [En ligne], 2017-2 | 2018, mis en ligne le 10 mars 2018, consulté le 10 décembre 2020. URL:

http://journals.openedition.org/itineraires/3787; DOI : https://doi.org/10.4000/itineraires.3787

Ce document a été généré automatiquement le 10 décembre 2020.

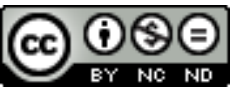

Itinéraires est mis à disposition selon les termes de la licence Creative Commons Attribution - Pas d'Utilisation Commerciale - Pas de Modification 4.0 International. 


\title{
Faire naître et mourir les vagues : comment s'écrit l'histoire des féminismes
}

How Waves Arise and Die Out: The Writing of the History of Feminism

\author{
Bibia Pavard
}

\section{Introduction}

1 L'histoire des mobilisations féministes s'écrit maintenant sur plusieurs siècles (Offen 2012). Après l'émergence de voix individuelles et collectives revendiquant l'égalité entre les sexes dans des contextes révolutionnaires depuis la fin $\mathrm{du}$ XVIII ${ }^{\mathrm{e}}$ siècle, les premières organisations revendiquant de nouveaux droits pour les femmes émergent au mitan du $\mathrm{XIX}^{\mathrm{e}}$ siècle, à peu près simultanément dans différents contextes occidentaux en voie de démocratisation. C'est donc sur le temps long qu'il faut penser les mouvements féministes, leurs continuités et leurs renouveaux. La métaphore marine s'est désormais imposée pour distinguer une première vague féministe se déployant $\mathrm{du} \mathrm{XIX}^{\mathrm{e}}$ siècle aux années 1930 autour de la question des droits civils et civiques, d'une deuxième vague émergeant dans les années 1960 davantage centrée sur la lutte des femmes contre le patriarcat et pour les libertés. Cette métaphore a été réactivée alors que l'on parle depuis les années 1990 d'une troisième vague (Henry 2004, Dean 2009) et même plus récemment d'une quatrième vague depuis les années 2010 qui serait portée par l'activisme en ligne ${ }^{1}$.

2 La vague, dans l'imaginaire politique, évoque la puissance d'un mouvement qui balaye l'ensemble de la société pour la transformer en profondeur. Comme l'écrit la féministe et pacifiste Marcelle Capy dans le premier numéro du périodique La vague en 1918, la «vague féminine »:

[...] vient des chantiers, des ateliers, des écoles, des campagnes; elle monte de partout où les corps des femmes sont accablés, où les cœurs des femmes sont brisés. Elle monte du peuple féminin qui halète sur les machines, pâlit sur les registres; $d u$ peuple féminin qui a faim, qui a froid, qui pleure, qui pense. [...] Elle monte à 
l'assaut de l'injustice sociale, des préjugés, des erreurs, de la violence érigée en dogme.

3 En histoire politique et culturelle, la «nouvelle vague " évoque aussi le renouveau générationnel ; que l'on songe à la célèbre enquête du magazine L'Express commandée à l'IFOP sur les « 18-30 ans ", lancée dans son numéro du 3 octobre 1957 et qui est restée dans la postérité grâce à un livre du même titre de Françoise Giroud publié en 1958 et au mouvement cinématographique du même nom. Néanmoins, les générations féministes ont leur existence propre et des temporalités distinctes des générations politiques et intellectuelles étudiées par les historiens (Sirinelli 1989). Réfléchir à la manière dont l'histoire des féminismes s'écrit permet de contribuer à la réflexion sur les temporalités, entre le temps long et la rupture des événements, sur les conventions qui conduisent à « découper l'histoire en tranches » (Le Goff 2014) et sur la diversité des récits historiques au gré des déplacements de regards (Zancarini-Fournel 2008 et 2016). La métaphore des ressacs féministes met l'accent à la fois sur les temporalités et sur l'intensité : il y a des vagues et des creux de la vague, des moments forts pendant lesquels la vague se forme et déferle et des moments de calme après la tempête qui peuvent être associés à des retours de bâton ou backlash (Faludi 1991). Dans l'histoire des féminismes, la métaphore en est aussi venue à distinguer des mouvances et dans cette acception les vagues s'additionnent : comme l'explique Christine Bard « plutôt que d'imaginer une succession des vagues, chacune chassant l'autre, il faut plutôt observer que lorsque la mer monte, les vagues se chevauchent, la plus neuve gagnant du terrain » (Bard 2017).

4 Pourtant cette terminologie, dès son apparition, suscite de nombreux débats chez les historien'ne's et les spécialistes des mouvements sociaux qui l'utilisent tout en démontrant ses limites. En effet, comment articuler le temps et l'espace de mobilisations qui se déploient tant au niveau local, national, régional qu'international selon des chronologies décalées ? Comment rendre compte des continuités autant que des moments de rupture ? Comment évoquer la diversité et des rapports de domination (de classe, de race, etc.) dans les mobilisations avec une métaphore unificatrice ? Cet article se propose, dans une perspective généalogique, de retracer les développements et les critiques de la métaphore des vagues féministes dans le contexte français avec une attention particulière aux phénomènes de circulations transnationales. À travers cette histoire, il s'agit de montrer comment les vagues naissent et sont transformées précisément à des moments de renouvellements générationnel et théorique et qu'il s'agit d'un instrument pour définir les contours d'un mouvement et d'un champ d'études. Il s'agira de faire une histoire des usages politiques et académiques de la métaphore des vagues, pour en déceler les liens et les écarts. La métaphore est donc ici l'objet même de l'analyse pour souligner comment elle a évolué et changé de sens depuis son installation dans les années 1970.

\section{Naissance des vagues (années 1960-1980) ou le retour des pionnières}

5 La première vague et la deuxième vague sont des notions qui se consolident en même temps, au tournant des années 1960 et 1970 dans le contexte étasunien. Comme l'explique l'historienne américaine Astrid Henry :

À la fin des années 1960 et au début des années 1970, les féministes commencèrent à

s'identifier comme la «seconde vague» (second wave) du mouvement des femmes 
tout en désignant parallèlement la période entre 1848 (la Convention de Seneca

Falls) et 1920 (l'obtention du vote) comme la « première vague ». (Henry $2004: 58$ )

L'occurrence initiale du terme de seconde vague serait médiatique, il s'agirait d'un article de la journaliste Martha Weinman Lear dans le New York Time Magazine en mars $1968^{2}$. Des militantes du mouvement féministe l'emploient ensuite à la fois pour s'inscrire dans la continuité des luttes précédentes mais aussi pour s'en distinguer, "dans une lecture positiviste des générations fondée sur l'idée de progrès selon laquelle chaque génération est comprise comme allant plus loin que la précédente ${ }^{3}$ » (Henry $\left.2004: 59-60\right)$. Le terme de seconde vague est par exemple utilisé par un groupe féministe bostonien pour nommer son journal publié de 1971 à 1983 : Second Wave: A Magazine of the New Feminism ${ }^{4}$. La militante Shulamith Firestone, pionnière du mouvement féministe new-yorkais explique que «d'un point de vue féministe radical, le nouveau féminisme n'est pas simplement la renaissance d'un important mouvement pour l'égalité sociale. C'est la seconde vague de la révolution la plus populaire de l'histoire ${ }^{5}$ ». Kate Millet, quant à elle, dans sa thèse intitulée Sexual Politics en 1970 veut voir advenir une «seconde vague de la Révolution sexuelle » (Studer et Thébaud 2004).

7 En France, la métaphore des vagues n'accompagne pas le renouveau féministe de la fin des années 1960 et du début des années 1970, car l'idée de rupture est beaucoup plus affirmée dans ce moment d'autodéfinition et de qualification de l'histoire en train de se faire. Une collection de textes rédigés en français et traduits de l'anglais est publiée sous le titre «Libération des femmes années zéro » dans la revue Partisan de juillet-septembre 1970. Ce titre évoque un commencement et fait table rase du passé. Il s'agit d'annoncer l'apparition d'un nouveau mouvement féministe révolutionnaire distinct du mouvement ouvrier, par les femmes et pour les femmes, luttant contre leur oppression spécifique, comme l'affirme un autre texte fondateur du mouvement « Combat pour la libération de la femme » publié dans L'Idiot international en mai 1970 par Monique et Gille Wittig, Marcia Rothenburg et Margaret Stephenson (Collectif 2009). En effet, le mouvement de libération des femmes marque une rupture tant dans la manière de s'organiser (refus de leaders, de l'institutionnalisation, groupes informels et spontanés) que dans les revendications (avortement, lutte contre le sexisme, le viol, les violences) et les modes d'action (happenings, humour, manifestations festives, réunions) (Chaperon 2000 : 377-378).

Pour autant, les années 1970 voient aussi se développer un « souci du passé » de la part des féministes autour d'un " "paradigme du continent noir", c'est-à-dire une critique de l'histoire comme récit (que ce soit l'histoire scientifique ou l'histoire servant de base à l'élaboration du roman national) sous l'angle de la mise en évidence de ses "oublis", de ses "silences", de "son continent noir" » (Charpenel 2014 : 163). Ce souci se manifeste par les références à des figures du passé, militantes ou mythiques qui participent à la lutte des femmes à travers les âges : sorcières persécutées, citoyennes tricoteuses, pétroleuses pendant la Commune, etc. (Ibid.). Néanmoins, la connaissance parcellaire du passé des combats féministes, surtout orientée vers le mouvement ouvrier, ne permet pas de parler de première ou seconde vague: les mouvements actifs pour les droits des femmes et notamment le droit de vote à la fin du XIX ${ }^{\mathrm{e}}$ siècle et au début du XX $\mathrm{X}^{\mathrm{e}}$ siècle sont inconnus des jeunes militantes des années 1970. Il faut attendre le développement de l'histoire des femmes à partir de 1973, en lien étroit avec le mouvement féministe, pour voir progresser les savoirs.

Les premiers travaux universitaires sur les féminismes des $\mathrm{XIX}^{\mathrm{e}}$ et $\mathrm{XX}^{\mathrm{e}}$ siècles en France datent des années 1980 mais n'utilisent pas la métaphore des vagues. Il s'agit de défricher 
un vaste terrain encore vierge et de forger des repères temporels et conceptuels. Laurence Klejman et Florence Rochefort, alors en thèse d'histoire sur le féminisme de la III ${ }^{e}$ République, donnent quelques «éléments d'une histoire du féminisme en France » dans le premier numéro de la revue Matériaux pour l'histoire de notre temps en 1985 :

Se discernent deux temps dans l'histoire du féminisme, encore à enrichir. Dans un premier temps le féminisme est le fruit de quelques réflexions individuelles et largement d'expression littéraire. Le deuxième temps débute avec la Révolution française qui marque une coupure dans la forme revêtue par le féminisme qui s'organise en mouvement collectif, politique, inaugurant ainsi une période qui perdure. (Klejman et Rochefort $1985: 6$ )

L'enjeu est surtout de se défaire d'une histoire du féminisme qui avait depuis l'entredeux-guerres été écrite sur le temps très long, depuis l'Antiquité (Abensour 1921, Albistur et Armogathe 1977) pour faire émerger la spécificité de la période contemporaine. Ce sont les historiennes américaines travaillant sur l'hexagone qui emploient la métaphore, comme Karen Offen, qui fait le point sur « l'état des connaissances sur le féminisme de la première vague en France » en 1982. Quant au premier travail sur les années 1970 en France, celui de Claire Duchen en 1986 en anglais, il précise qu'« il y a de profondes différences entre le MLF et les féministes qui précèdent, mais il y a aussi de nombreuses continuités, des expériences similaires, des problèmes et des questions partagées par le “nouveau" et l'“ancien" féminisme ${ }^{6}$ " (Duchen 1986 : 4). Les débats historiographiques en France portent plutôt sur la définition du féminisme et sur les catégorisations (féminisme radical, réformiste, etc.) (Thébaud 1998: 92). En 1995, un numéro des Cahiers du CEDREF (Centre d'enseignement, de documentation et de recherches pour les études féministes) de l'université Paris 7 est consacré aux " continuités et discontinuités du féminisme » et interroge les "féminismes dans l'histoire », « la fragilité des acquis » et pose la question de comment «transmettre aujourd'hui » révélant les liens étroits entre connaissance de l'histoire et action politique (autour de l'avortement et la parité). Si la question de l'héritage et des générations affleure, la métaphore des vagues n'apparaît pas centrale. Un article de Christine Bard, cependant, consacré à la sclérose des associations dans les années 1930, présente «la crise du féminisme en France dans les années 1930 » et rappelle que «depuis deux cents ans, sur un thème commun, de nouvelles variations surgissent, entrecoupées de silences» (Bard 1995). Paradoxalement la première thèse d'histoire qui popularise la métaphore des vagues, celle de Sylvie Chaperon, l'utilise de façon critique pour s'intéresser au « creux de la vague », les années 1945-1970 (Chaperon 1996). Elle rejoint ainsi un ensemble de travaux qui, en approfondissant les connaissances sur les mouvements féministes, invitent à prendre des distances avec la métaphore marine.

\section{De l'importance des creux}

11 Dès les années 1980, des travaux académiques en anglais critiquent la notion de vague. Verta Taylor, notamment, a travaillé sur la continuité du mouvement féministe à partir d'une étude des mouvements pour les droits des femmes aux États-Unis de 1945 aux années 1960. Dans son article de 1989, traduit en français en 2005 sous le titre « La continuité des mouvements sociaux. La mise en veille du mouvement des femmes ", elle explique ses réticences. Se fondant sur la théorie des opportunités politiques, elle décrit des processus de mise en veille dans les mouvements sociaux dans des contextes qui ne sont pas favorables à leur expression et l'existence d'organisations dormantes (en anglais 
abeyance Structures) qui vont assurer le lien entre deux moments favorables au développement de mouvements: en assurant la survie des réseaux militants, en conservant une identité collective et en faisant vivre un répertoire de buts et de tactiques notamment. Elle a ainsi étudié le parti national des femmes (NWP), branche du mouvement des suffragettes aux EU, sa survie après 1945 et les passerelles qui existent avec l'Organisation nationale pour les femmes (National organisation for women, NOW) fondée en 1966 qui est une des organisations centrales de la "seconde vague ». Elle en conclut :

La plupart des mouvements connaissent des seuils ou des points de retournements que les chercheurs ont bien souvent confondus avec des mouvements de naissance ou de mort. Je suggère ici que les mouvements ne meurent pas, mais se rétractent et se retranchent pour s'adapter aux changements de climat politique. Peut-être les mouvements ne naissent-ils jamais réellement. (Taylor 2005 : 250)

La période que Verta Taylor étudie pour les États-Unis a été étudiée pour la France par Sylvie Chaperon. Elle montre elle aussi la vitalité de cet entre-deux où certes les associations nées au tournant $\mathrm{du} \mathrm{xx}^{\mathrm{e}}$ siècle sont vieillissantes mais perdurent et où émerge une nouvelle génération de militantes qui ne se revendique pas du féminisme mais poursuit des combats autour des droits civils, des droits des femmes au travail et se concentre aussi sur le nouveau combat du contrôle des naissances faisant le lien avec la génération suivante des années 1968.

Certaines chercheuses proposent d'autres métaphores qui seraient plus parlantes. Karen Offen, qui est l'auteure d'un ouvrage à la chronologie atypique puisqu'il étudie les féminismes en Europe de 1750 à 1950 préfère la métaphore volcanique : elle parle de "féminisme en termes d'éruption, de coulée, de fissures de lave en fusion (de magma), car [elle] le considère plutôt comme une forme menaçante et insaisissable de mécontentement, qui ne cesse de s'attaquer aux points faibles des couches de sédiments accumulés qui forment la cuirasse du patriarcat, le vernis institutionnel des sociétés organisées (et réussit parfois à se frayer un chemin au travers) ( (Offen 2012 : 56). Cette métaphore insiste davantage sur un magma continu et une croûte terrestre qui cède à certains moments, dans certaines circonstances.

Ces différents travaux montrent qu'à mesure que les recherches universitaires sur les mouvements féministes s'étoffent, la réalité décrite apparait de plus en plus multiple et difficilement réductible. Paradoxalement c'est en «creux » qu'elle s'impose d'abord, les flux et reflux se révélant limités pour penser ensemble des connaissances de plus en plus nombreuses et complexes. Pourtant, avec les renouveaux militants des années 1990 et les transformations du champ universitaire sur les femmes, la métaphore des vagues connaît une deuxième vie malgré les critiques dont elle a fait l'objet.

\section{La troisième vague : du marqueur temporel au marqueur identitaire (années 1990 et 2000)}

Après ce que Susan Faludi a nommé le backlash des années 1980 (Faludi 1991) - période somme toute assez courte - les années 1990 correspondent à un renouveau du militantisme féministe autour de courants et groupes constitués par des militant·e's née's dans les années 1970, n'ayant pas connu le MLF. Ce renouveau militant se caractérise par la poursuite de certaines revendications et l'émergence de nouvelles (le droit au mariage pour les personnes de même sexe, les droits pour les personnes trans, la parité, etc.) et de 
nouvelles solidarités transnationales (après la conférence de l'ONU de Pékin en 1995). Le contexte politique a changé, la fin de la guerre froide et la chute de l'URSS sont accompagnées d'un fort discrédit du marxisme qui était la matrice idéologique des années 1968. En outre, la fin des années 1990 et le début des années 2000 sont marqués par « l'exacerbation des conflits politico-religieux, du conflit israélo-arabe notamment, et l'attentat du 11 septembre 2001 [qui] n'ont fait que renforcer les oppositions, réelles ou supposées, entre émancipations et religions, et le clivage entre les Nords et les Suds » (Rochefort 2010: 1090). Le contexte intellectuel est, lui aussi, marqué par le postmodernisme. Les années 1980 et 1990 ont correspondu à l'institutionnalisation des études sur les femmes, le féminisme puis le genre à l'université (Lagrave 1990, Thébaud 1998, Andriocci 2005). L'émergence et la diffusion de la théorie queer notamment portée par la philosophe américaine Judith Butler et son essai Gender Trouble en 1990 ont irrigué tant la pensée que l'action en dénaturalisant encore davantage les identités sexuées comprises comme des performances et en proposant de ne plus fonder le féminisme a priori sur le sujet femme (Bessin et Dorlin 2005). Une nouvelle génération de militante-s désormais appelés LGBT (lesbiennes, gays, bis, trans) participe largement au renouveau féministe en prônant la convergence des luttes. Ce sont ces nouvelles associations qui se retrouvent dans les théories queer qui, à partir des années 1990, rétablissent « une certaine osmose entre mouvement homosexuel et mouvement féministe " après les dissensions des années 1970 et 1980 (Chauvin $2005:$ 131).

C'est dans ce contexte que le terme de troisième vague se forge, une fois encore aux ÉtatsUnis. L'historienne Astrid Henry (2015), rappelle que le terme est notamment popularisé par Rebecca Walker dans son article publié en 1992 dans Ms Magazine intitulé «Becoming the third wave ». Née en 1969, elle est la fille d'Alice Walker, l'écrivaine afro-américaine reconnue (elle a notamment écrit $\mathrm{La}$ Couleur pourpre) et la filleule de Gloria Steinem (militante féministe blanche fondatrice de Ms Magazine). Dans cet article elle explique que le combat est loin d'être terminé et elle écrit : «Je me sens prête à décider, comme ma mère l'a fait, de consacrer la majeure partie de mon énergie à l'histoire, la santé et le soin des femmes. Tous mes choix devront soutenir mes principes féministes de justice ${ }^{7}$ »; elle conclut: "Je ne suis pas une féministe postféministe. Je suis la troisième vague. » Son utilisation marque une continuité avec la vague précédente puisqu'elle s'oppose à l'idée impliquée par le postféminisme, que celui-ci n'est plus nécessaire. Néanmoins, elle s'inscrit en rupture par rapport à la génération précédente, réflexion poursuivie dans son ouvrage Being real: Telling the Truth and Changing the Face of Feminism. Elle dénonce le dogmatisme militant précédent et plaide pour un féminisme qui accepte la complexité des identités individuelles (elle est elle-même bisexuelle et bi-raciale) et prenne en compte d'autres combats que la lutte des femmes. Son texte reçoit beaucoup d'écho et en 1994 elle est nommée parmi les cinquante leaders les plus influents dans Time magazine. Elle est à l'origine de la Third wave foundation. Selon Astrid Henry, Rebecca Walker est représentative d'une nouvelle génération de féministes, qui s'engagent individuellement ou dans des groupes à partir des années 1990 et qui se considèrent comme les filles de la génération précédente. Cette nouvelle génération est plus «mixte racialement » et plus ancrée dans les questionnements sur les identités de genre et les identités sexuelles multiples dans une approche intersectionnelle. De même, Jonathan Dean, spécialiste du féminisme britannique contemporain, note que le terme de troisième vague lorsqu'il émerge à la fin des années 1980 et au début des années 1990 au Royaume-Uni renvoie à deux réalités. Premièrement il s'agit d'un positionnement politique et théorique en 
rupture avec les féminismes des années 1970, perçu comme essentialisant un sujet femme monolithique. À partir d'un positionnement post-structuraliste et post-colonial, il s'agit de promouvoir un féminisme plus inclusif. Deuxièmement, il s'agit de décrire une nouvelle génération de militantes revendiquant une nouvelle forme de féminisme (Dean 2009). La métaphore des vagues change alors de signification. Elle semble s'émanciper de l'idée de "vaste mouvement " pour qualifier une somme d'appropriations individuelles, et de l'idée de "moment historique » pour devenir marqueur identitaire d'ordre générationnel et politique. La troisième vague sert à distinguer les mères des filles tandis que la deuxième vague séparait des pionnières du passé de nouvelles pionnières issues des mouvements révolutionnaires des années 1960. Les deux manières de comprendre la métaphore, selon des enjeux chronologiques ou des enjeux théorético-politiques, existent chez les militantes et les militants (Evans 2015). Cette acception plurielle de la métaphore permet d'annoncer l'arrivée d'une quatrième vague n'ayant plus rien à voir avec un vaste mouvement survenu après un reflux mais qui désigne un changement de contexte: le regain d'intérêt pour le féminisme dans les médias et la culture de masse dans les années 2010 (Rivers 2017).

17 En France, les années 1990-2000 correspondent également à des formes de remobilisation mais la métaphore de la troisième vague n'est pas utilisée. En 1995, la grande manifestation de défense du droit à l'avortement réunit 40000 personnes et aboutit à la création du Collectif national pour les droits des femmes (CNDF), pour coordonner l'action des associations féministes mais aussi des "groupes femmes", de partis politiques et de syndicats. Ce collectif organise les 15 et 16 mars 1997 des Assises nationales pour les droits des femmes qui réunissent deux mille personnes (Henneron 2005). De plus, «la bataille de la parité » en politique fait converger des individus, des groupes et des associations de différents horizons politiques et de différentes générations (Bereni 2015). Cette mobilisation implique des militantes actives depuis les années 1970 dans le MLF, des militantes des partis politiques de gauche, des militantes d'associations féminines plus traditionnelles et des intellectuelles. De plus, l'institutionnalisation du féminisme permet une coordination des actions dans l'État et en dehors de l'État. L'aboutissement de ce mouvement est le changement de la constitution en 1999 et le vote d'une loi en 2000 visant à assurer l'égal accès des hommes et des femmes aux fonctions politiques. Objectivement, cette intense mobilisation pour l'égalité des sexes et son succès s'inscrivent pleinement dans le renouveau du féminisme. Pourtant, les «jeunes féministes » qui se mobilisent au même moment ne s'y retrouvent pas et affirment leur différence, sans toutefois employer le terme de troisième vague.

Les militantes féministes actives dans les années 1990-2000 appartiennent en effet à différentes cohortes démographiques: celles qui ont débuté leur engagement dans les années 1968 sont toujours actives tandis que d'autres née's dans les années 1970 et 1980 les ont rejointees. Au-delà de la différence d'âge, il y a aussi des différences politiques : "Deux générations et deux manières différentes de militer coexistent aujourd'hui dans le mouvement, chaque génération apportant un renouvellement des formes du féminisme ", écrit la sociologue Liane Henneron (2005 : 95). Il y avait aussi différentes cohortes actives dans les années 1970, ce que montre l'analyse des signataires du manifeste des 343 femmes ayant déclaré avoir avorté dans Le Nouvel Observateur le 5 avril 1971, puisqu'il est par exemple signé par Simone de Beauvoir (née en 1908), Gisèle Halimi (née en 1927) et des militantes du MLF nées dans les années 1940 et 1950 (Pavard 2012). Néanmoins, la coexistence générationnelle n'est pas comparable dans la mesure où les féministes des 
années 1970 ne côtoient pas un mouvement féministe antérieur mais plutôt quelques individualités et quelques groupes. En revanche, les jeunes qui commencent à militer dans les années 1990 et 2000 font face à une génération au sens fort de "génération historique», c'est-à-dire de rencontre entre "quelques cohortes démographiques proches » et un "événement fondateur de grande amplitude » qui forge une identité commune (Sirinelli 1989 : 73). Il existe en effet une " génération MLF », terme qui dépasse largement les seules militantes de ce mouvement et qui qualifie la spécificité politique et culturelle de la rencontre entre les filles du baby-boom et les « années 1968 » (Artières et Zancarini-Fournel 2008). Il existe donc bien "différentes générations militantes" (Henneron 2005), mais comment qualifier la nouvelle génération militante des années 1990-2000?

Des associations se créent à la fin des années 1990 et au début des années 2000, comme les «Marie pas claires » qui tiennent un journal de 1992 à 1999, les «Sciences potiches se rebellent » au sein de Sciences Po en 1995, « Mix-cité » en 1997, « Ni putes ni soumises » et «Les Panthères roses » (association LGBT et féministe) en 2003. Elles sont fondées par de jeunes militantes et militants qui négocient leur rapport aux féministes des années 1970. La transmission se produit dans le cadre Familial (Henneron 2005, Masclet 2015), militant ou parfois universitaire (Andriocci 2005, Bereni 2012a). Néanmoins, comme l'explique Liane Henneron, il s'agit de créer de nouvelles structures militantes, soit par méconnaissance des structures existantes, soit par volonté de se réunir entre jeunes. Ces groupes revendiquent de nouveaux modes d'organisation : la plupart de ces associations (sauf les « Marie pas claires ») accueillent des hommes et rompent ainsi avec la théorisation politique de la non-mixité des années 1970, jugée passéiste, la mixité étant brandie comme un marqueur générationnel (Jacquemart 2015 : 113-115). De plus, ces groupes revendiquent des modes d'action radicaux, inspirés du happening et tournés contre ou vers les médias et des manifestations festives, rompant ainsi avec les modes d'action des associations issues des années 1980 plus centrés sur l'influence sur les pouvoirs publics, l'expertise ou le travail social (Henneron $2005: 105$, Herman 2016). Pour autant l'héritage n'est pas forcément rejeté, et en cela le parcours de Clémentine Autain est révélateur. Née en 1973, de parents artistes et engagés à gauche, elle milite à l'UNEF et à l'UEC pendant ses études d'histoire à l'université Paris 8. À la suite d'un viol elle s'engage dans le "Collectif féministe contre le viol», créé en 1985 et animé par des militantes de longue date, et elle réalise son DEA sur «Mouvement social, féminisme et législation à travers l'exemple du corps des femmes (1967-1982)». Elle co-fonde ensuite l'association « Mix-cité » qui est reçue dans les médias comme un signe de renouveau du militantisme féministe. D'autres groupes marquent plus clairement une rupture avec les féministes des années 1970. Les militant·es. de « Ni putes ni soumises » qui revendiquent leur origine dans les cités reprochent ainsi aux féministes pré-existantes d'avoir oublié les milieux populaires.

L'apparition de ces groupes donne lieu à des articles qui annoncent un nouveau féminisme. Les médias participent à l'affirmation d'une rupture politique et générationnelle. Par exemple, en 1995, un article de Libération annonce : « Zarmazones et Marie pas claires réinventent la lutte. Choisissant les rythmes funk ou l'humour, elles redonnent un coup de jeune à la cause des femmes ${ }^{8}$.» Les années 1990 et le début des années 2000 voient ainsi se poser la question de la transmission dans le mouvement féministe dans la mesure où ces associations au départ ne se disent pas féministes ( «Mixcité » se dit antisexiste) et s'inscrivent en rupture. Au milieu des années 2000, le problème 
de la transmission revient très souvent sur le devant des débats entre les groupes féministes (Henneron 2005). Sabine du groupe «Les Marie pas claires » évoque dans une interview en 1995 les relations difficiles avec la génération précédente :

On a beaucoup de mal à faire des choses ensemble; on les appelle «les vieilles " quand elles nous énervent, on a un rapport conflictuel qui est un rapport de génération à la con. Elles ont du mal à nous accepter parce qu'elles ont du mal à nous cerner; elles ont tellement eu l'habitude de militer toutes seules, d'être dans la galère, d'avoir connu un grand mouvement puis son reflux que du coup, elles ont du mal à croire qu'on représente quelque chose. Soit elles essayent de nous utiliser en disant, "venez nous faire rigoler 5 minutes ", soit elles nous oublient, elles font comme si on n'existait pas9.

21 La rupture générationnelle est renforcée à la fin de la décennie 2000, moment où plusieurs facteurs se conjuguent. Tout d'abord, de nouvelles associations se créent ( La Barbe » en 2008 ; « Osez le féminisme » en 2009 ; « Les Femen » en 2008 en Ukraine et 2012 en France), elles sont animées par de «nouvelles jeunes» qui ne sont pas de la même génération militante que celle qui avait porté le renouveau des années 1990. Les Femen focalisent particulièrement l'attention des médias et se revendiquent porteuses d'un militantisme qu'elles dénomment "sextremisme ${ }^{10}$ ", en rupture avec les "vieilles féministes ». Ensuite, le développement de l'usage militant du web contribue à renforcer les différences générationnelles face à l'appropriation de ces outils en mettant sur le devant de la scène des jeunes femmes qui maitrisent les codes de la communication numérique (Fotopoulou 2014, Jouët, Niemeyer et Pavard 2017).

Enfin, la rupture n'est pas seulement générationnelle elle est aussi politique. Les fortes divergences autour de la loi de 2004 sur l'interdiction des signes religieux ou sur la pénalisation de la prostitution ont renforcé les clivages. Le développement de groupes féministes qui se disent queer, inclusifs, afroféministes ou musulmans renforce l'idée que la troisième vague correspond à une mouvance plus soucieuse de la reconnaissance de la diversité sociale, raciale, sexuelle ou religieuse (Lamoureux 2006). La qualification « de la troisième vague » en vient alors parfois à signifier la démarche de "mise en question du sujet politique du féminisme » (Noyé 2017) dans une approche intersectionnelle qui met en avant les rapports de pouvoir traversant le groupe des femmes. L'autodéfinition du collectif « 8 mars pour toutes » créé en 2012 est révélatrice de ce type de positionnement.

Nous estimons qu'il n'y a pas un mais DES féminismes, et qu'aucune vision du féminisme ne peut s'ériger en modèle universel. Le collectif se définit donc comme féministe non-excluant, favorisant la prise de parole et l'action des premièrEs concernéEs par les violences sexistes. Chaque communauté, chaque minorité en lutte contre les violences sexistes est porteuse de revendications différentes face à des oppressions multiples, et c'est à elles de déterminer comment s'en libérer ${ }^{11}$.

Le renouveau militant n'est pas porteur du retour de la métaphore en France même si elle apparaît çà et là notamment dans l'ouvrage de 2004 sur Le Siècle des féminismes (Gubin et al. 2004). En revanche la commémoration des quarante ans de 1968 vient donner une cohérence à la "deuxième vague ${ }^{12}$ " tandis que des travaux universitaires, qui s'inscrivent dans le calendrier mémoriel, s'approprient pleinement la métaphore des vagues. 


\section{Les vagues féministes " entre histoire et mémoire » (Studer et Thébaud 2004)}

24 Marion Charpenel, qui a réalisé une thèse de sociologie politique sur la mémoire féministe, a montré à quel point les commémorations étaient omniprésentes pendant la période de son terrain entre 2005 et 2010 avec un climax entre 2008 et 2010. La célébration de l'anniversaire du MLF cristallise l'attention et les tensions. Elle voit d'abord l'affrontement de groupes militants autour de la date de naissance du MLF (1968 ou 1970) et la création d'une association pour organiser les quarante ans du MLF en 2010. Cette célébration est un moment d'« élaboration et de diffusion d'une lecture dominante du passé » par certaines militantes engagées depuis les années 1970 (Charpenel 2014 : 361). C'est autour de cette commémoration que l'on voit s'affirmer le terme de féministes historiques pour qualifier les militantes actives depuis les années 1970. Marion Charpenel note l'existence de ce terme dans les interactions observées; il vise à légitimer la participation au comité d'organisation des quarante ans du MLF au titre d'un engagement pérenne (Charpenel $2014: 378$ ). Il se diffuse aussi dans les médias et sert à marquer les différences générationnelles. Par exemple dans un article de l'AFP du 6 mars 2008, on peut lire: «Qu'en est-il du mouvement féministe 40 ans après mai 1968 et la radicalité subversive du MLF ? La journée des Femmes du 8 mars a-t-elle encore un sens ? Pour les féministes "historiques", les jeunes doivent inventer une autre forme de militantisme, pour "défendre des acquis menacés"13."

Les « 40 ans du MLF » sont aussi l'occasion pour les historien-ne-s de produire des travaux. Entre 2008 et 2010, plusieurs séminaires, journées d'études et colloques sont organisés pour rendre compte de l'avancée des connaissances sur les féminismes des années 1968, construire des archives et susciter de nouveaux travaux ${ }^{14}$. Ces événements donnent lieu à des publications qui questionnent la chronologie. L'introduction du numéro spécial de la revue Clio, histoire et genre, résultat d'un colloque tenu à Lyon en 2008, précise ainsi :

Dire que 1968 a été le moment de la «révolution sexuelle» fait partie des lieux communs sur la période, comme d'affirmer que la «deuxième vague» du mouvement féministe est née en 1970. Dans ce numéro de Clio HFS, nous revisitons les chronologies, les formes et les lieux de mobilisation collective - en faisant jouer les jeux d'échelle du local au national -, pour comprendre comment des « individue-s ordinaires " se sont saisis des revendications et ont subverti les normes de genre. (Pohrel et Zancarini-Fournel 2009 : § 5)

C'est aussi l'occasion d'intégrer quasi systématiquement le terme de féminisme de la deuxième vague. L'expression est alors utilisée tantôt pour qualifier une période (les années 1968) tantôt pour signifier la nécessité de dépasser le seul MLF dans l'étude des «années Mouvement» (1970-1981) (Picq 1993). Par exemple, dans l'ouvrage tiré du colloque consacré aux «Féministes de la $2^{\mathrm{e}}$ vague, actrices du changement social» les 20-22 mai 2010 à Angers, l'introduction de Christine Bard précise que «le féminisme le plus visible est celui qui revendique son autonomie, dans des associations qui se proclament indépendantes. Mais il ne s'agit que de la partie émergée de l'iceberg - ou du volcan» (Bard 2012: 13). L'ouvrage s'ouvre ainsi sur l'article de Laure Bereni conceptualisant «l'espace de la cause des femmes» défini comme la « configuration des sites de mobilisation pour la cause des femmes dans une pluralité de sphères sociales " (Bereni 2012b : 28) et invitant à penser la transversalité des mouvements sociaux au-delà de la notion réductrice de « mouvement féministe ». 
Ces publications témoignent aussi d'effets d'âge dans le champ académique, puisqu'elles rassemblent trois générations de chercheuses sur l'histoire des féminismes : celle qui a vécu les années 1970 et qui a écrit sur la période, telle Françoise Picq (1993) et qui préserve sa mémoire; celle qui a donné les premières thèses sur l'histoire du féminisme des XIX et début XXe siècle à la fin des années 1980 et au début des années 1990 (Christine Bard, Sylvie Chaperon, Florence Rochefort, etc.) et enfin celle qui vient d'achever ou est en thèse lors de cette commémoration (dont je fais partie) et qui travaille sur le XIX comme sur le $\mathrm{xx}^{\mathrm{e}}$ siècle, jusqu'à la période très contemporaine en histoire, sociologie ou science politique. Cette nouvelle génération de chercheuses qui débute au tournant des années 1990-2000 bénéficie des travaux précédents, des quelques cours ou séminaires existants sur le sujet à l'Université, et des directeurs et directrices de thèses spécialistes du sujet. Nombreuses appartiennent à l'association Efigies qui réunit depuis 2003 les jeunes chercheuses et chercheurs sur le genre, le féminisme et les sexualités. Comme l'a bien montré Laure Bereni :

[...] le renouvellement générationnel perceptible à partir du tout début des années 2000 s'est poursuivi sans discontinuer depuis lors: de nouvelles et nouveaux entrants ont gonflé les rangs de cette nouvelle vague, apportant leurs propres problématiques théoriques et politiques, redéfinissant les frontières et le sens de ce renouvellement générationnel. (Bereni 2012a)

Les thèses sur l'histoire du féminisme soutenues dans les années 2000 insistent encore davantage sur la pluralité des mobilisations, sur les formes de circulation en s'inscrivant dans une démarche critique des concepts de la sociologie des mouvements sociaux tout en utilisant la métaphore des vagues (Bereni et Revillard 2012). On peut émettre l'hypothèse que des logiques propres au champ académique - notamment la possibilité d'enseigner l'histoire du féminisme et la nécessité de le rendre intelligible à des jeunes publics - a contribué à normaliser la métaphore des vagues et ses vertus pédagogiques. Elle fait ainsi son entrée dans le Dictionnaire des féministes en France (Bard et Chaperon 2017).

Pourtant, paradoxalement, alors que la métaphore des vagues est admise comme outil dans les travaux académiques récents, elle est aussi mise à distance comme objet. Beaucoup de spécialistes des féminismes se sont ainsi exprimés dans les médias ou les cercles académiques pour relativiser la nouveauté du «nouveau féminisme » et inciter à l'inscription des mobilisations actuelles dans le temps plus long de l'histoire des féminismes. Christine Bard a ainsi comparé les usages militants du corps des Femen et des suffragettes anglaises au tournant $\mathrm{du} \mathrm{xx}^{\mathrm{e}}$ siècle (Bard 2014) et des travaux invitent par exemple à replacer le militantisme en ligne dans le temps long des rapports entre féminisme, médias et communication (Blandin 2017, Pavard 2017). Certaines vont plus loin et recommandent de ne pas l'utiliser, comme un collectif d'historiennes des femmes américaines qui «ont le mal de mer » et s'interrogent: «Est-il temps de sauter du bateau?» (Laughlin et al. 2010 : 76). Pour elles, la métaphore des vagues favorise une vision unifiée du féminisme, un "féminisme hégémonique " centré sur des actrices blanches hétérosexuelles et de classe moyenne-supérieure alors que les travaux historiques montrent que les "pratiques féministes sont apparues et ont perduré dans des formes très différentes et dans des contextes sociaux, politiques et culturels variés " (Laughlin et al. 2010 : 78). De fait les travaux actuels sur le mouvement lesbien dans les années 1970-1980, par exemple, n'obéissent pas à la même chronologie qui fait des années 1980 un moment de reflux (Eloit 2017). On pourrait ajouter que la métaphore des vagues se concentre sur certaines zones géographiques (Amérique du Nord, Europe de 
l'Ouest et du Nord, Australie), laissant de côté d'autres aires où les mobilisations féministes se déploient selon des temporalités distinctes par exemple en Europe de l'Est, Afrique, Amérique du Sud, Asie, monde arabe (Badran 2009, Cîrstocea et Giraud 2015). La focale est aussi sur l'échelle nationale, laissant de côté les mobilisations locales parfois décalées dans le temps (Godard et Porée 2014 ; Masclet 2017), c'est pourquoi Christine Bard préconise une réflexion sur la "territorialisation » des études sur les féminismes pour prendre du recul par rapport à l'échelle nationale (Bard 2017).

\section{Conclusion : une métaphore pour écrire l'histoire ou pour la faire?}

Malgré ses limites, la métaphore des vagues a une histoire qui s'insère dans les contextes politique et intellectuel des féminismes depuis les années 1970. Elle a une utilité politique, permettantde décrire une mobilisation jaillissante avec des spécificités nouvelles tout en rendant compte d'un long passé de luttes. La figure de style est en phase avec l'idée révolutionnaire d'une déferlante militante qui doit faire table rase du passé. Elle a aussi une utilité académique : celle de délimiter les périmètres des recherches émergentes sur différentes périodes historiques et donc de coller aux spécialisations des historien·ne·s, dix-neuviémistes, ou vingtiémistes. Ses usages se sont donc construits à la croisée entre les enjeux militants et les enjeux académiques, ce qui fait varier le sens qu'on lui donne. Tantôt évocation des temporalités des mobilisations, la métaphore des vagues permet de délimiter des périodes et des contextes, démarche utile pour penser l'action, écrire l'histoire et l'enseigner. Tantôt évocation des continuités et des ruptures elle permet de poser la question de la transmission et du renouvellement générationnel dans le champ militant. Tantôt synonyme de mouvance politique, elle sert à insister sur la diversité des mobilisations féministes à un temps $\mathrm{T}$. Cette extrême fluidité entre apport descriptif et portée prescriptive montre à quel point il s'agit d'une catégorie à la fois indigène et savante, c'est sa faiblesse mais c'est aussi sa force.

\section{BIBLIOGRAPHIE}

Abensour, Léon, 1921, Histoire générale du féminisme : des origines à nos jours, Paris, Librairie Delagrave.

Albenga, Viviane et Jacquemart, Alban, 2015, « Pour une approche microsociologique des idées politiques », Sociétés contemporaines, $\mathrm{n}^{\circ}$ 109, p. 7-20.

Albistur, Maïté et Armogathe, Daniel, 1977, Histoire du féminisme en France du Moyen Âge à nos jours, Paris, Les éditions des femmes.

Andriocci, Muriel, 2005, « Entre colère et distance : les “études féministes” à l'université ", L'Homme et la société, n 158, p. 73-93. 
Artières, Philippe et Zancarini-Fournel, Michelle, 2008, 68. Une histoire collective, Paris, La Découverte.

Badran, Margot, 2009, Feminism in Islam, Oxford, Oneworld.

Bard, Christine, 1995, « La crise du féminisme en France dans les années trente », Les cahiers du CEDREF, $\mathrm{n}^{\circ} 4-5$, p. 13-27, [En ligne], http://journals.openedition.org/cedref/291, consulté le 9 mars 2018.

Bard, Christine (dir.), 2012, Les Féministes de la deuxième vague, Rennes, PUR.

Bard, Christine, 2014, " "Mon corps est une arme”, des suffragettes aux Femen », Les Temps Modernes, $\mathrm{n}^{\circ} 678$, p. 213-240.

Bard, Christine, 2017, « Faire des vagues », dans K. Bergès, F. Binard et A. Guyard-Nedelec (dir.), Féministes au XXI ${ }^{e}$ siècle : une troisième vague?, Rennes, PUR, p. 31-45.

Bereni, Laure, 2012a, « Une nouvelle génération de chercheuses sur le genre. Réflexions à partir d'une expérience située ", Contretemps, [En ligne], https://www.contretemps.eu/une-nouvellegeneration-de-chercheuses-sur-le-genre-reflexions-a-partir-dune-experience-situee/, consulté le 9 janvier 2017.

Bereni, Laure, 2012b, « Penser la transversalité des mobilisations féministes : l'espace de la cause des femmes ", dans C. Bard (dir.), Les Féministes de la deuxième vague, Rennes, PUR, p. 27-41.

Bereni, Laure et Revillard, Anne, 2012, « Un mouvement social paradigmatique, ce que le mouvement des femmes fait à la sociologie des mouvements sociaux ", Sociétés contemporaines, $\mathrm{n}^{\circ}$ 85, p. 17-42.

Bereni, 2015, La Bataille de la parité. Mobilisations pour la féminisation du pouvoir, Paris, Économica. Bessin, Marc et Dorlin, Elsa, 2005, « Les renouvellements générationnels du féminisme : mais pour quel sujet politique ? ", L'Homme et la société, n 158 , p. 11-27.

Blandin, Claire, 2017, « Le web, de nouvelles pratiques militantes dans l'histoire du féminisme ? ", Réseaux, vol. 35, nº 201, p. 9-17.

Boris, Eileen, Cobble, Dorothy Sue, Gallagher, Julie et Laughlin, Kathleen A., 2010, « Is It Time to Jump Ship? Historians Rethink the Waves Metaphor », Feminist Formations, vol. 22, n 1, p. 76-135. Chaperon, Sylvie, 1996, Le Creux de la vague. Mouvements féminins et féministes 1945-1970, Thèse de l'institut universitaire européen, département d'histoire et civilisation.

Chaperon, Sylvie, 2000, Les Années Beauvoir (1945-1970), Paris, Fayard.

Charpenel, Marion, 2014, «Le privé est politique! » Sociologie des mémoires féministes en France, Thèse de doctorat de science politique de l'Institut d'études politiques de Paris.

Chauvin, Sébastien, 2005, «Les aventures d'une "alliance objective”. Quelques moments de la relation entre mouvements homosexuels et mouvements féministes au $\mathrm{xx}^{\mathrm{e}}$ siècle ", L'Homme et la société, $\mathrm{n}^{\circ} 158$, p. 111-133.

Cîrstocea, Ioana et Giraud, Isabelle, 2015, « Pluralisme des mouvements féministes contemporains ", L'Homme et la société, nº 198.

Clair, Isabelle et Heinen, Jacqueline, 2013, « Le genre et les études féministes françaises : une histoire ancienne. Introduction », Cahiers du Genre, n 54, p. 9-19.

Collectif, 2009, MLF//Textes premiers, Paris, Stock. 
Dean, Jonathan, 2009, "Who's Afraid of Third Wave Feminism? On the uses of the "Third Wave" in British Politics », International Feminist Journal of Politics, vol. 11, nº 3, p. 334-352.

Dorlin, Elsa (dir.), 2008, Black feminism. Anthologie du féminisme africain-américain 1975-2000, Paris, L'Harmattan.

Duchen, Claire, 1986, Feminism in France from May 68 to Mitterrand, Londres, Routledge, Kegan Paul. Eloit, Ilana, 2017, « "Le bonheur était dans les pages de ce mensuel”. La naissance de la presse lesbienne et la fabrique d'un espace à soi (1976-1990) », Le Temps des Médias, n² 29, p. 93-108.

Evans, Elizabeth, 2015, « What Makes a (Third) Wave? How and Why the Third Wave Narrative Works for Contemporary Feminists », International Feminist Journal of Politics, n 18, p. 409-428.

Faludi, Susan, 1991, Backlash. The Undeclared War against American Women, New York, Crown. Firestone, Shulamith, [1970] 1993, The Dialectic of Sex. The Case for Feminist Revolution, New York, Farrar, Straus and Giroux.

Fotopoulou, Aristea, 2014, « Digital and Networked by Default? Women's Organisations and the Social Imaginary of Networked Feminism », New Media \& Society, vol. 18, p. 989-1005.

Gallot, Fanny, 2015, En découdre. Comment les ouvrières ont révolutionné le travail et la société, Paris, La Découverte.

Gallot, Fanny et Meuret-Campfort, Ève, 2015, « Des ouvrières en lutte dans l'après 1968. Rapports au féminisme et subversions de genre ", Politix, n 109, p. 21-43.

Godard, Patricia et Porée, Lydie, 2014, Les femmes s'en vont en lutte. Histoire et mémoire du mouvement féministe à Rennes (1965-1985), Rennes, Goater.

Gubin, Éliane, Jacques, Catherine, Rochefort, Florence, Studer, Brigitte, Thébaud, Françoise et Zancarini-fournel, Michelle (dir.), 2004, Le Siècle des féminismes, Paris, L’Atelier.

Henneron, Liane, 2005, « Être jeune féministe aujourd'hui : les rapports de génération dans le mouvement féministe contemporain ", L’Homme et la société, $\mathrm{n}^{\circ}$ 158, p. 93-111.

Henry, Astrid, 2004, Not my mother's sister. Generational Conflict and Third-Wave Feminism, Blomington, Indiana University Press.

Henry, Astrid, 2015, « From a mindset to a movement. Feminism since 1990 », dans D. Sue Cobble, L. Gordon et A. Henry, Feminism Unifinished, New York, Liveright.

Herman, Elisa, 2016, Lutter contre les violences conjugales. Féminisme, travail social et politiques publiques, Rennes, PUR.

Hypathia, 1997, vol. 12, n 3, «Third wave feminisms ».

Jacquemart, Alban, 2015, Les Hommes dans les mouvements féministes. Socio-histoire d'un engagement improbable, Rennes, PUR.

Jouët, Josiane, Niemeyer, Katharina et Pavard, Bibia, 2017, « Faire des vagues. Les mobilisations féministes en ligne ", Réseaux, vol. 35, n² 201, p. 21-57.

Klejman, Laurence et Rochefort, Florence, 1985, «Éléments d'une histoire du féminisme en France ", Matériaux pour l'histoire de notre temps, $\mathrm{n}^{\circ} 1$, p. 6-19.

Lagrave, Rose-Marie, 1990, « Recherches féministes ou recherches sur les femmes », Actes de la recherche en sciences sociales, $\mathrm{n}^{\circ} 83, \mathrm{p} .27-39$.

Lamoureux, Diane, 2006, "Y a-t-il une troisième vague féministe ? ", Cahiers du Genre, $\mathrm{n}^{\circ}$ 3, (HS n ${ }^{\circ}$ 1), p. 57-74. 
Laughlin, Kathleen A., 1990, « Is It Time to Jump Ship? Historians Rethink the Waves Metaphor », Feminist Formations, vol. 22, $\mathrm{n}^{\circ} 1$, p. 76-135.

Le Goff, Jacques, 2014, Faut-il vraiment découper l'histoire en tranches ?, Paris, Seuil.

Masclet, Camille, 2015, « Le féminisme en héritage ? Enfants de militantes de la deuxième vague », Politix, $n^{\circ}$ 109, p. 45-68.

Masclet, Camille, 2017, Sociologie des féministes des années 1970. Analyse localisée, incidences biographiques et transmission familiale d'un engagement pour la cause des femmes en France, Thèse de doctorat de sociologie de l'université de Lausanne et de l'université Paris 8.

Noyé, Sophie, 2017, « Troisième vague », dans C. Bard et S. Chaperon, Dictionnaire des féministes, France XVIII ${ }^{e}-\mathrm{XXI}^{e}$ siècle, Paris, PUF.

Offen, Karen, 1982, « "First Wave” Feminism in France: New Work and Ressources », Women studies international forum, vol. 5, $\mathrm{n}^{\circ}$ 6, p. 685-689.

Offen, Karen, 2012, Les Féminismes en Europe, 1700-1950, Rennes, PUR.

Pavard, Bibia, 2012, Si je veux, quand je veux. Contraception et avortement dans la société française (1956-1979), Rennes, PUR, coll. « Archives du féminisme ».

Pavard, Bibia, 2017, « Les mobilisations féministes en France à l'ère d'internet : pour une approche sociohistorique », dans K. Bergès, F. Binard, A. Guyard-Nedelec (dir.), Féministes au XXI siècle : une troisième vague?, Rennes, PUR, p. 161-174.

Picq, Françoise, 1993, Libération des femmes. Les années Mouvement, Paris, Seuil.

Porhel, Vincent et Zancarini-Fournel, Michelle, 2009, Clio. Histoire, femmes et sociétés, $\mathrm{n}^{\circ} 29$, « 68', révolutions dans le genre?», [En ligne], http://journals.openedition.org/clio/9165.

Revillard, Anne, 2016, La Cause des femmes dans l'État. Une comparaison France-Québec, Grenoble, Presses universitaires de Grenoble.

Riot-Sarcey, Michèle, 1994, La Démocratie à l'épreuve des femmes. Trois figures critiques du pouvoir, Désirée Véret, Eugénie Niboyet et Jeanne Deroin 1830-1848, Paris, Albin Michel.

Riot-Sarcey, Michèle, 2002, Histoire du féminisme, Paris, La Découverte.

Rivers, Nicola, 2017, Postfeminism(s) and the Arrival of the Fourth Wave. Turning Tides, Londres, Palgrave MacMillan.

Rochefort, Florence, 2010, « Troisième vague féministe, religions et sécularisations, 1990-2007 ", dans C. Fauré (dir.), Nouvelle encyclopédie politique et historique des femmes, Paris, Les Belles Lettres, p. 1096-1114.

Rochefort, Florence et Zancarini-Fournel, Michelle, 2005, « Du féminisme des années 1970 aux débats contemporains ", dans M. Maruani (dir.), Femmes, genre et sociétés : l'état des savoirs, Paris, La Découverte, p. 347-355.

Sirinelli, Jean-François, 1989, « Génération et histoire politique », Vingtième Siècle. Revue d'histoire, $\mathrm{n}^{\circ} 22$, p. 67-80.

Studer, Brigitte et Thébaud, Françoise, 2004, « Entre histoire et mémoire », dans É. Gubin, C. Jacques, F. Rochefort, B. Studer, F. Thébaud et M. Zancarini-fournel (dir.), Le Siècle des féminismes, Paris, L'Atelier, p. 27-45.

Taylor, Verta, 2005, « La continuité des mouvements sociaux. La mise en veille des mouvements de femmes », dans O. Fillieule (dir.), Le Désengagement militant, Paris, Belin, p. 229-250. 
Thébaud, Françoise, 1998, Écrire l'histoire des femmes, Lyon, ENS éditions.

Zancarini-Fournel, Michelle, 2008, Le Moment 68. Une histoire contestée, Paris, Seuil.

Zancarini-Fournel, Michelle, 2016, Les luttes et les rêves. Une histoire populaire de la France, 1685 à nos jours, Paris, La Découverte.

\section{NOTES}

1. Sur cette question, voir Kira Cochrane, "The Fourth Wave of Feminism: Meet the Rebel Women ", The Guardian, 10 décembre 2013, [En ligne], https://www.theguardian.com/ world/2013/dec/10/fourth-wave-feminism-rebel-women ; Claire Touzard, "Se dirige-t-on vers un nouveau féminisme ? », Grazia, 8 avril 2016, http://www.grazia.fr/article/se-dirige-t-on-versun-nouveau-feminisme-811024; et pour un regard plus académique Ealasaid Munro, « Feminism: A Fourth Wave?», Political Insight, vol. 4, n 2, 2013, p. 22-25, [En ligne], http:// journals.sagepub.com/doi/full/10.1111/2041-9066.12021.

2. «In short, feminism, which one might have thought as dead as the Polish Question, is again an issue; Proponents call it the Second wave, the First having ebbed after the glorious victory of suffrage disappeared, finally into the great sandbar of Togertherness. » Voir Astrid Henry (2004 : 58).

3. "On the one hand, the metaphore of the wave allowed feminists to make connections across time, enabling women to see themselves as part of an ongoing movement for women's rights ans equality; On the other hand, this metaphor has often relied on a positivist understanding of generations founded on the idea of progress in which each generation is understood to go beyond the generation which came before it. "

4. The Second Wave: A Magazine of the New Feminism records, http://www.lib.neu.edu/archives/ collect/findaids/m19find.htm

5. «In the radical feminist view, the new feminism is not just the revival of a serious political movement for social equality. It is the second wave of the most popular revolution in history " (Shulamith Firestone [1970] 1993 : 15).

6. «There are profound differences between the MLF and previous feminisms, but there are also many continuities, similar experiences, problems and issues shared by "new" and “old" feminism."

7. «I am ready to decide, as my mother decided before me, to devote much of my energy to the history, health, and healing of women. Each of my choices will have to hold to my feminist standard of justice. [...] I am not a postfeminism feminist. I am the Third Wave. »

8. Marie Colmant et Cécile Daumas, Libération, 25 novembre 1995, [En ligne] http:// next.liberation.fr/vous/1995/11/25/zarmazones-et-marie-pas-claire-reinventent-la-luttechoisissant-les-rythmes-funk-ou-l-humour-elles-r_148876.

9. Les Marie pas claire et Anne Dhoquois, « Nouvelle génération, nouvelles féministes? ", Les cahiers du CEDREF, nº 4-5, 1995, [En ligne], http://journals.openedition.org/cedref/305.

10. «Femen, les militantes au seins nus ouvrent un centre d'entraînement au féminisme à Paris », AFP, 18 septembre 2012: «La France a beaucoup d'organisations féministes classiques mais rien qui représente le nouveau féminisme », a-t-elle dit, définissant ce dernier comme du «+sextrémisme+, du terrorisme pacifique ».

11. Collectif 8 mars pour toutes, page Facebook, onglet «À propos»/ «Histoire ", https:// www.facebook.com/pg/Collectif8MarsPourToutes/about/?ref=page_internal, consulté le 15 septembre 2017.

12. Deuxième vague remplace alors seconde vague puisque dorénavant il y en a une troisième. 
13. Martine Veron, «Les petites filles de Mai 68 à la recherche d'un nouveau féminisme », AFP, 6 mars 2008.

14. Groupe de recherche sur les féminismes 2007-2008, EFIGIES/CMH-ETT: «Les féministes de l'après 68 en France [...] prêtant une attention particulière aux mobilisations féministes qui ont émergé à la suite de mai 68. Ces mobilisations ont marqué le renouvellement du militantisme féministe et ont constitué un moment crucial dans l'émergence du féminisme dit de la "Deuxième vague", tout autant qu'un aspect fondamental des années 1968. »

\section{RÉSUMÉS}

La métaphore des vagues s'est imposée dans les années 1970 pour décrire l'histoire des mobilisations féministes depuis le $\mathrm{XIX}^{\mathrm{e}}$ siècle en Occident. Elle est en même temps de plus en plus contestée dans le milieu académique depuis que les débats sur l'existence d'une troisième vague sont apparus dans les années 1990. Cet article se propose de faire un bilan des usages académiques et militants de cette métaphore afin de leur redonner une historicité et de montrer le poids des enjeux politiques dans ces définitions. Il s'agit, à travers le cas de la France, de montrer comment la métaphore des vagues témoigne de la circulation des appellations entre le discours militant et le discours académique et révèle les tensions existantes pour savoir qui décide de faire naître et mourir les vagues.

The "waves" metaphor, which emerged in 1970s to describe the history of feminist mobilizations in the West, has been increasingly challenged in the academic field since the 1990s, when discussions of the "third wave" of feminism emerged. This article reviews the academic and activists' uses of the metaphor, providing both a historical account and deeper understanding of its political implications. A case study of the wave metaphor in France highlights the international circulations of designations between the activist and the academic discourse. It reveals the existing tensions around what activities or initiatives are included in the various waves, which bodies are present, and when the waves arise or die.

\section{INDEX}

Mots-clés : féminisme, vagues féministes, histoire du féminisme, historiographie, générations

Keywords : feminism, feminist waves, generations, history of feminism, France

\section{AUTEUR}

\section{BIBIA PAVARD}

Université Paris 2 Panthéon-Assas, CARISM (EA 2293) 